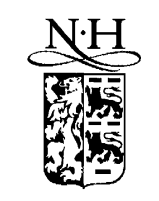

ELSEVIER

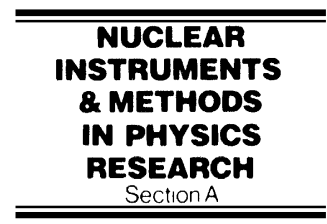

www.elsevier.com/locate/nima

\title{
Simulations of the TJNAF FEL with tapered and inversely tapered undulators
}

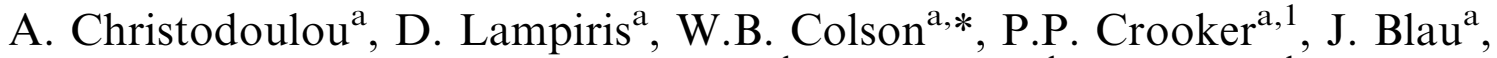 \\ R.D. McGinnis ${ }^{\mathrm{a}}$, S.V. Benson ${ }^{\mathrm{b}}$, J.F. Gubelib, G.R. Neil ${ }^{\mathrm{b}}$ \\ ${ }^{a}$ Physics Department, Naval Postgraduate School, 833 Dyer Road, Monterey, CA 93943, USA \\ ${ }^{\mathrm{b}}$ FEL Department, Thomas Jefferson National Accelerator Facility, Newport News, VA 23606, USA
}

\begin{abstract}
Experiments using the TJNAF FEL have explored the operation with both tapered and inversely tapered undulators. We present here numerical simulations using the TJNAF experimental parameters, including the effects of taper. Singlemode simulations show the effect of taper on gain. Multimode simulations describe the evolution of short optical pulses in the far infrared, and show how taper affects single-pass gain and steady-state power as a function of desynchronism. A short optical pulse presents an ever-changing field strength to each section of the electron pulse so that idealized operation is not possible. Yet, advantages for the recirculation of the electron beam can be explored. (C) 2001 Elsevier Science B.V. All rights reserved.
\end{abstract}

PACS: $41.60 \mathrm{Cr}$

Keywords: Taper; Inverse taper; Free-electron laser; Simulation

\section{Introduction}

For any free-electron laser (FEL), the undulator design determines the physics of the interaction between the relativistic electrons and the copropagating light pulse [1]. One way to alter the undulator design is to introduce taper. Taper may be achieved by varying the magnetic field along the undulator, and it has been shown that positively and negatively tapered undulators have characteristics that can be advantageous for particular

\footnotetext{
*Corresponding author. Tel.: +1-831-656-2765; fax: +1831-656-2834.

E-mail address: colson@nps.navy.mil (W.B. Colson).

${ }^{1}$ On leave from Department of Physics and Astronomy, University of Hawaii, Honolulu, HI 96822, USA.
}

applications in strong optical fields [2-8]. Recently, Thomas Jefferson National Accelerator Facility (TJNAF) has experimentally explored many properties of the same FEL with no taper, positive taper, and negative taper [9]. The purpose of this paper is to numerically simulate those experiments with a view to theoretically understanding and extending the TJNAF experiments.

\section{The TJNAF free-electron laser}

The TJNAF FEL has $N=41$ undulator periods of length $\lambda_{0}=2.7 \mathrm{~cm}$ each. It was operated with short 0.5 ps electron pulses (length $l_{\mathrm{e}}=150 \mu \mathrm{m}$ ) of total energy $E=34.5 \mathrm{MeV}$ with a $0.25 \%$ energy 
spread, $I=50 \mathrm{~A}$ peak current, $\lambda=6 \mu \mathrm{m}$ optical wavelength, and a resonator $Q=10$. The dimensionless undulator parameter $K=e B_{\text {rms }} \lambda_{0} / 2 \pi m c^{2}=0.98$, with linear taper rates of $\Delta K / K= \pm 5 \%, \pm 7.5 \%$, and $\pm 10 \%$. From these values, we characterize the TJNAF FEL using dimensionless parameters: the current $j=8 N\left(\pi e K N \lambda_{0}\right)^{2} \rho F / \gamma^{3} m c^{2}=10$, where $\rho$ is the beam electron density, and the electron pulse length $\sigma_{z}=l_{\mathrm{e}} / N \lambda=1$. The taper is contained in the pendulum equation torque $\delta=-\left[4 \pi N K^{2} /\left(1+K^{2}\right)\right](\Delta K / K)=0, \pm 4 \pi, \pm 6 \pi$, $\pm 8 \pi$; and for a cavity of length $S$, the desynchronism is given by $d=-\Delta S / N \lambda$ and is varied from 0 to 0.4 .

\section{Single mode results}

Single mode behavior was explored by examining the gain for a range of initial phase velocities $v_{0}$ and initial fields $a_{0}$. It is interesting here to contrast the general properties of positive and negative taper. For positive taper, the optimum phase velocity in strong fields is at resonance $\left(v_{0}=0\right)$ where bunching occurs for electrons initially trapped in the closed orbit region of phase space. For negative taper, the optimum phase velocity is above resonance $\left(v_{0}>0\right)$, where bunching occurs as electrons travel around the closed orbit region of phase space. The motivation for using either positive or negative taper is to improve performance in strong fields $\left(a_{0} \gg \pi\right)$.

Fig. 1 shows the gain plotted against both the initial optical field $a_{0}$ and the initial phase velocity $v_{0}$ for the cases of $\delta= \pm 8 \pi$ and $j=10$. For reference, the untapered ( $\delta=0$, not shown) weakfield $\left(a_{0} \lesssim \pi\right)$ gain peaks at $G=176 \%$, and decreases to $12 \%$ in strong fields $\left(a_{0} \gtrsim \pi\right)$ [1]. As the taper increases, the curves are initially shifted along the $v_{0}$ axis by $-\delta / 2$, the weak-field peak is reduced, and the strong field peak is increased. At large taper, as shown in Fig. 1, the gain spectrum becomes highly distorted. For $\delta=+8 \pi$, the single $\delta=0$ peak is replaced by two comparably sized peaks, which decrease from $29 \%$ in weak fields to $17 \%$ in strong fields. Two comparably sized peaks also appear for $\delta=-8 \pi$, but in that case the peaks do not merge at strong fields, decreasing from $27 \%$ in weak fields to $17 \%$ in strong fields. In strong fields the gain with either taper is better than that with no taper. Also note that the two curves in Fig. 1 are related to each other in the sense that $G\left(v_{0}, \delta, a_{0}\right) \simeq-G\left(-v_{0},-\delta, a_{0}\right)$.

\section{Multimode results}

Multimode behavior is simulated by introducing a short parabolic electron pulse into the optical resonator and examining the evolution of the pulse and the optical mode as a function of the number of round trips $n$ the optical pulse has made through the resonator. Fig. 2 shows the results after 2000 passes through an undulator with positive taper $(\delta=+8 \pi)$. The upper graphs give the optical field shape $|a(z, n)|$, the optical power spectrum $P(v, n)$, and the electron spectrum $f(v, n)$ at the final pass. The shading in the middle graphs shows how these quantities have evolved with $n$. On the bottom left, the longitudinal profile of the current density $j(z-\tau)$ is shown for reference at dimensionless times $\tau=0$ and 1 . The bottom center graph shows the weak-field gain spectrum for reference, and the right-hand bottom graph shows the evolution of the total power $P$ as the pass number $n$ increases. Several parameters are printed across the top; the peak current $j$, the resonator $Q$, the pulse width $\sigma_{z}, \delta, N$, and the standard deviation of a small fluctuation $\delta \zeta$ in the initial electron phases. Also listed is the desynchronism $d=-\Delta S / N \lambda$, where $\Delta S$ is a reduction in resonator length from perfect synchronism. The displacement between the electron and optical pulses at $\tau=0$ on each pass is determined by $d$. In addition to showing many interesting details of the behavior, the simulations also print out the total steady-state power $P$ at saturation and the weak-field, steady-state gain $G$, both of which are strong functions of desynchronism. These quantities provide a good general assessment of the effect of taper on the FEL behavior as shown in Figs. 3 and 4.

Figs. 3 and 4 summarize the results of many simulations. The steady-state power $P$ and weakfield gain $G$ are plotted versus desynchronism for 


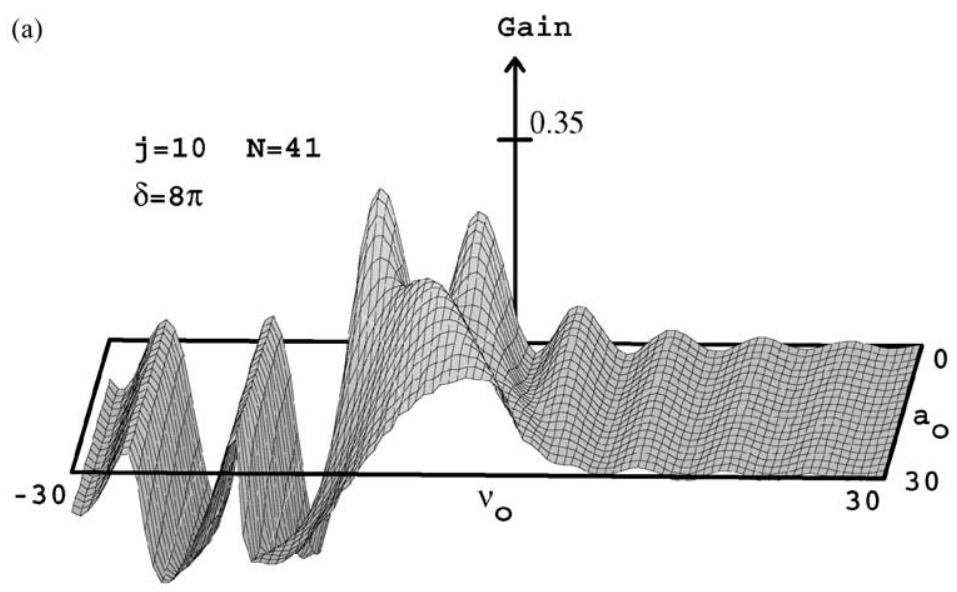

(b)

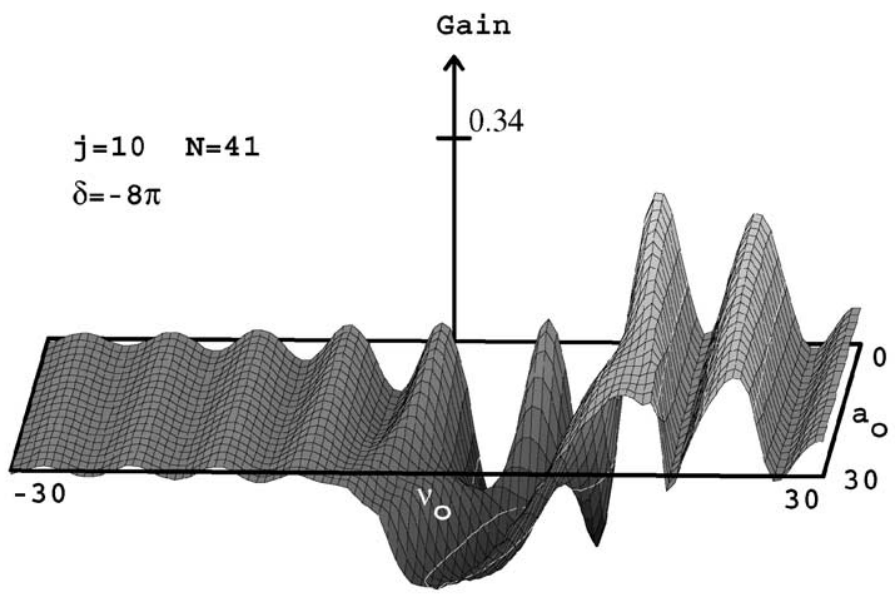

Fig. 1. FEL gain spectrum $G\left(v_{0}, a_{0}\right)$ for large tapers. (a) $\delta=+8 \pi$; (b) $\delta=-8 \pi$.

$\delta=0, \pm 4 \pi, \pm 6 \pi, \pm 8 \pi$ and $j=10$. The most general feature is that the operating range decreases as $\delta$ increases in magnitude. [The operating range is also a function of the current: a taper rate of $\delta=$ $\pm 8 \pi$ and $j=6$ (not shown) will not work at all.] For $d<0.004$ the laser will not operate, but the power rises sharply before $d \simeq 0.02$. Simultaneously, the weak-field gain increases. In the start-up region, the number of passes $n$ required to achieve the final power is large, but for higher $d$ the number of passes is greatly reduced. At small values of desynchronism, the optical pulse is short $(0.5 \mathrm{ps})$ with a broad spectrum. For $\delta=0$ and negative tapers, the sharp peaks in power are accompanied by evidence of the trapped-particle instability. This effect is reduced for positive tapers and vanishes for $\delta=8 \pi$. This instability is caused by electrons in the presence of strong optical fields becoming trapped in potential wells in phase space and oscillating at the trapped-particle synchrotron frequency $v_{\mathrm{s}}=\left(|a|^{2}-\delta^{2}\right)^{1 / 4}[1]$.

For larger $d$ and positive $\delta$, the power curves flatten, with the power diminishing significantly as the taper increases. For negative $\delta$, flattening does not occur and the power declines uniformly to zero with increasing $d$. In the same region, the steadystate gain is approximately parabolic for all tapers, but with reduced range in $d$ as $\delta$ increases in magnitude. Note that the untapered power exceeds the positively tapered power for most of the range 
of $d$. For $\delta=-4 \pi$, however, tapering provides power greater than the untapered case up to $d \simeq 0.18$, while for $\delta=-6 \pi$ the improvement ends at $d \simeq 0.09$. Finally, when $d$ is very large, the optical and electron pulses fail to overlap, and the power is reduced to zero. In that region, the

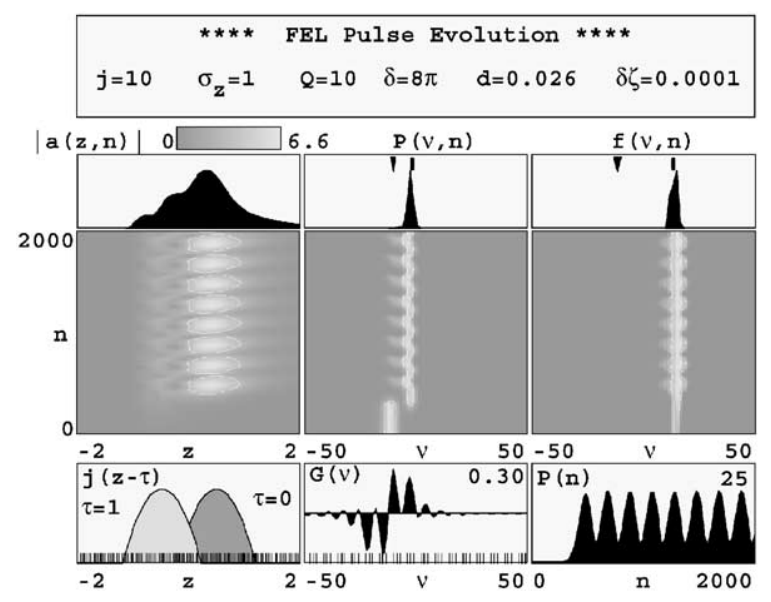

Fig. 2. Multimode simulation for $j=10, d=0.026$, and $\delta=$ $+8 \pi$. The various quantities are explained in the text. In this case, the oscillations in $|a(z, n)|, P(v, n), f(v, n)$, and $P(n)$ are evidence for limit-cycle behavior. optical pulses become very long, as much as $5 \sigma_{z}$, with a long leading edge. Note also that the gain is approximately the same for both positive and negative tapers.

For $d<0.1$, the final power, gain, and electron spectrum may oscillate regularly — up to $50 \%$ modulation of the average power in some cases and over hundreds of undulator passes. For these regions, shown by the large circles and squares on the power and gain curves in Figs. 3 and 4, only the peak values of the steady-state power and the average values of gain are shown. We attribute these non-steady effects to limit-cycle behavior, caused when trapped particles in strong fields combine with short optical pulses. The modulation, caused by the oscillation of the trapped current, continually modifies the shape of the short optical pulse. The different pulse shapes have different powers and spectra, causing oscillations as subpulse structures "march" through the pulse envelope. Fig. 2 shows an example for $\delta=+8 \pi$ and $d=0.026$, where one finds limit-cycle oscillations in $|a(z, n)|, P(v, n), f(v, n)$, and $P(n)$.

From the electron spectrum, we have also found the electron energy spread $\Delta \gamma / \gamma$ as a function of $d$ and $\delta$. The curves (not shown) appear much like the power curves of Figs. 3 and 4, with initial

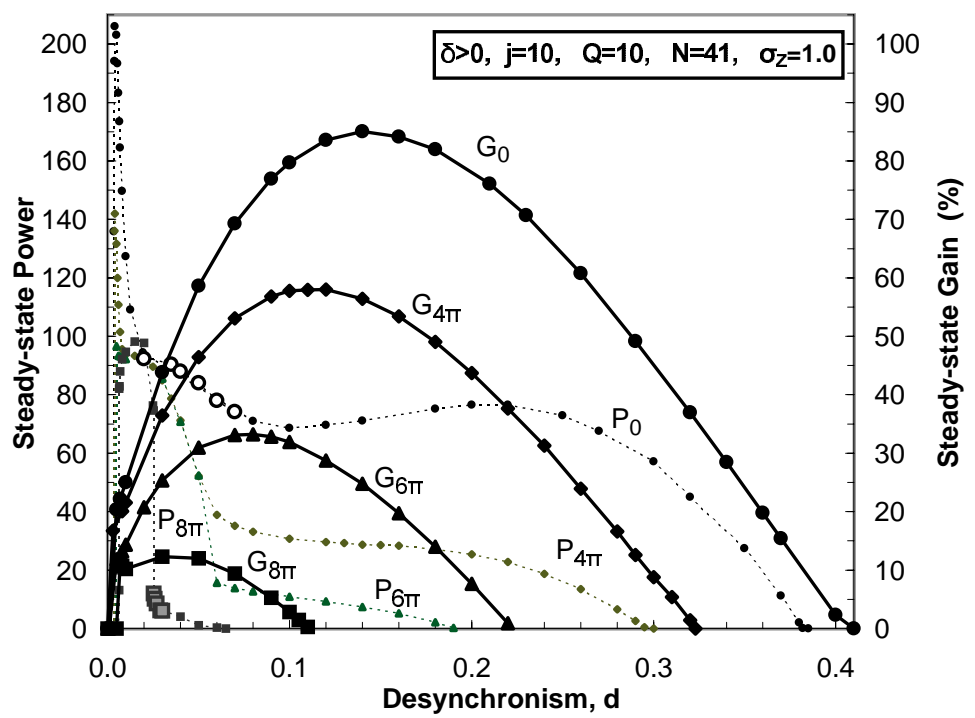

Fig. 3. Steady-state, saturated power $P$ and weak-field, steady-state gain $G$ versus desynchronism for positive taper rates $\delta=0,+4 \pi,+6 \pi$, and $+8 \pi$. 


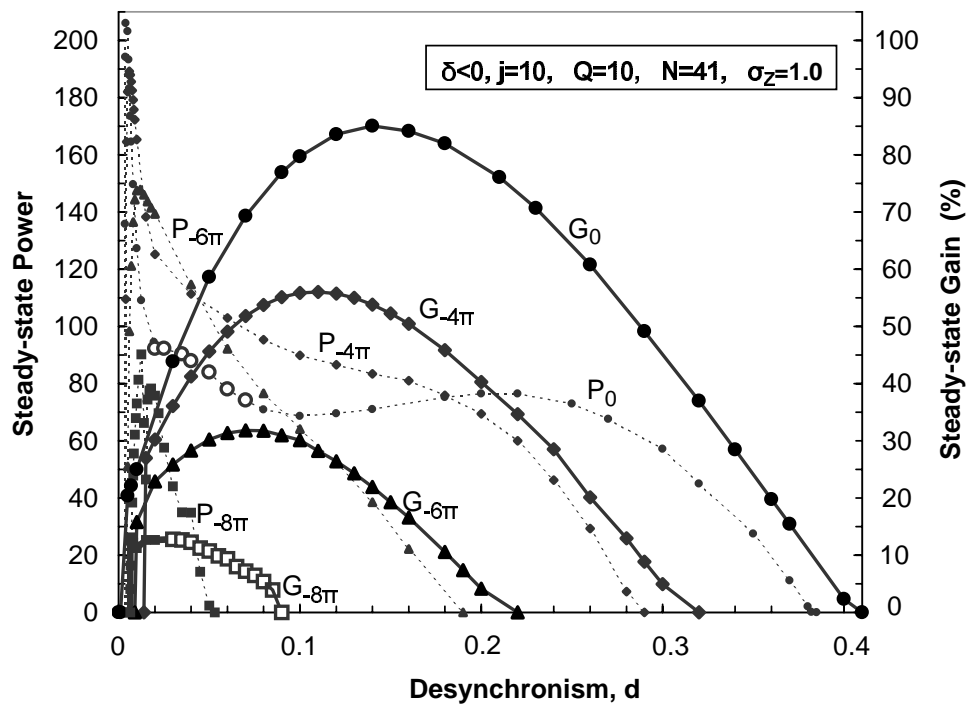

Fig. 4. Steady-state, saturated power $P$ and weak-field, steady-state gain $G$ versus desynchronism for negative taper rates $\delta=0,-4 \pi,-6 \pi$, and $-8 \pi$.

sharp peaks (up to $7 \%$ ) near $d=0.01$. For $d>0.05$ however, they are all less than $4 \%$.

Finally, when exploring these parameters, we find that at slightly larger values of $Q$ (above $Q=$ 12) and negative taper $(\delta=-8 \pi)$, gain oscillations are observed in weak fields over many passes. The optical pulse becomes spatially modulated at the slippage distance, but there are no trapped electrons. This is a new effect caused by mode competition between two competing peaks in the gain spectrum.

\section{Acknowledgements}

The authors are grateful for support by the Naval Postgraduate School.

\section{References}

[1] W.B. Colson, in: W.B. Colson, C. Pellegrini, A. Renieri (Eds.), Laser Handbook, Vol. 6, North-Holland, Amsterdam, 1990 (Chapter 5).
[2] G.R. Neil, S. Benson, G. Biallas, C.L. Bohn, D. Douglas, H.F. Dylla, R. Evans, J. Fugitt, J. Gubeli, R. Hill, K. Jordan, G. Krafft, R. Li, L. Merminga, D. Oepts, P. Piot, J. Preble, M. Shinn, T. Siggins, R. Walker, B. Yunn, First operation of an FEL in same-cell energy recovery mode, Proceedings of the 21st International FEL Conference, Hamburg, Germany, August, 1999. Nucl. Instr. and Meth. A 445 (2000) 192.

[3] N.M. Kroll, P.L. Morton, M.N. Rosenbluth, Physics of Quantum Electronics, Vol. 7, 89, 1980.

[4] D.A. Jaroszynski, R. Prazeres, F. Glotin, J.M. Ortega, Nucl. Instr. and Meth. A 358 (1995) 224.

[5] D.A. Jaroszynski, R. Prazeres, F. Glotin, J.M. Ortega, D. Oepts, A.F.G. van der Meer, G. Knippels, P.W. van Amersfoort, Nucl. Instr. and Meth. A 358 (1995) 228.

[6] D.A. Jaroszynski, R. Prazeres, F. Glotin, O. Marcouille, J.M. Ortega, D. Oepts, A.F.G. van der Meer, G. Knippels, P.W. van Amersfoort, Nucl. Instr. and Meth. A 375 (1996) 647.

[7] E.L. Saldin, E.A. Schneidmiller, M.V. Yurkov, Nucl. Instr. and Meth. A 375 (1996) 336.

[8] W.B. Colson, R.D. McGinnis, Nucl. Instr. and Meth. A 445 (2000) 49.

[9] S. Benson, J. Gubeli, G.R. Neil, Nucl. Instr. and Meth. A 475 (2001) 276, these proceedings. 